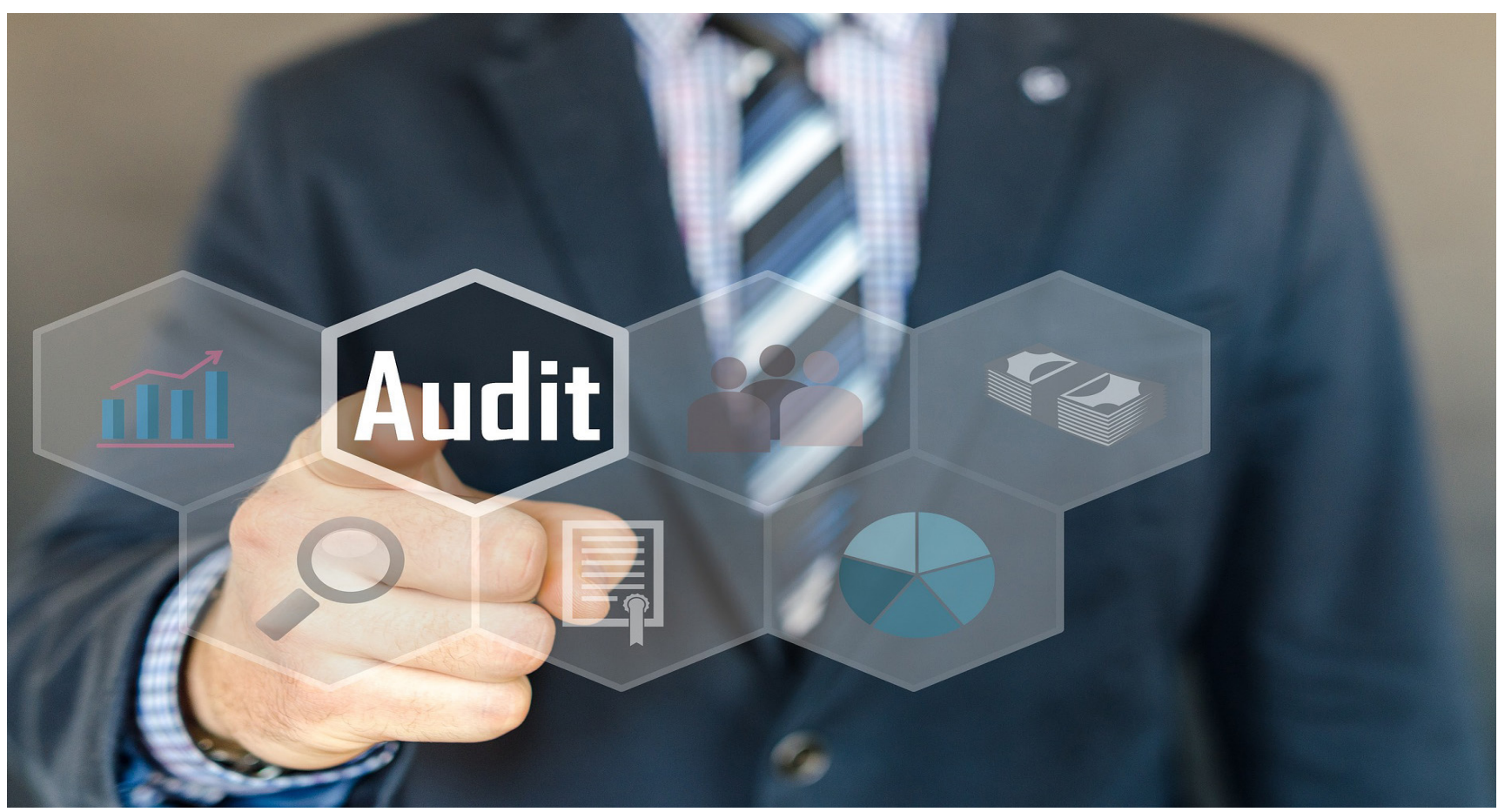

\title{
Perfil del Contador Público para el ejercicio de la auditoría financiera
}

\section{Qualities required by public accountants for the exercise of financial auditing}

Joseph Alejandro Suarez-Vera

Estudiantes del Programa de Contaduría Pública, Universidad Francisco de Paula Santander, Cúcuta, Colombia

\section{German Gustavo Ospina-Hernández}

Magister en Administración de Proyectos, gospina@ufps.edu.co,

Universidad Francisco de Paula Santander, Cúcuta, Colombia 


\section{Resumen}

El presente estudio tiene como objetivo determinar qué aspectos cualidades y características debe poseer el perfil de un contador público para el desarrollo de un encargo de auditoría financiera, para ello se realizó un proceso metodológico para la localización de los diferentes documentos bibliográficos que contribuyeran en el desarrollo del artículo en aspectos tales como definiciones, cualidades, propiedades, características entre otros, obteniendo que el perfil del contador público en el desarrollo de un encargo de auditoría financiera deberá de tener diferentes aspectos importantes tales como la imparcialidad, la diplomacia, la confidencialidad, el pensamiento crítico y la independencia, las cuales son útiles para el desarrollo de un auditoría financiera eficaz y eficientes, de esta forma concluyendo que un contador público que en su perfil cuente con alguna de estas características les ayudara en el desarrollo de cualquier proceso de auditoría, destacando que un auditor que posea estos aspectos en su perfil no será fácilmente influenciado por terceros para cometer adulteraciones en los informes de auditoría.

Palabras clave: Auditoria, Auditoría financiera, Competencias, Cualidades, Perfil del Auditor.

\section{Abstract}

The objective of this study is to determine which aspects, qualities and characteristics a public accountant's profile should possess for the development of a financial audit assignment. To this end, a methodological process was carried out to locate the different bibliographic documents that contributed to the development of the article in aspects such as definitions, qualities, properties, characteristics, among others, obtaining that the profile of the public accountant in the development of a financial audit assignment should have different important aspects such as impartiality, diplomacy, confidentiality, critical thinking and independence, which are useful for the development of an effective and efficient financial audit, thus concluding that a public accountant who has some of these characteristics in his profile will help them in the development of any audit process, highlighting that an auditor who has these aspects in his profile will not be easily influenced by third parties to commit adulterations in the audit reports.

Keywords: Audit, Auditor's Profile, Competencies, Financial Audit, Qualities. 


\section{4}

\section{Introducción}

La auditoría financiera ha ido evolucionando y mejorando con el tiempo con el propósito de examinar los estados financieros de una entidad de forma más adecuado, es por ende que según Álvarez (2015) "hoy en día es un elemento fundamental para la gerencia de los entes económicos, ya que les permite obtener una sistematicidad y obtener mejores resultados sobre su gestión administrativa" lo anterior se debe a que los resultados de una auditoria pueden ser usados por la gerencia para la toma de decisiones, por ende Álvarez denomina la auditoría financiera un elemento fundamental para la gerencia.

Por otra parte, se debe tener en cuenta que la auditoria varía dependiendo de cada auditor ya que según Gonzales, Alfaro, Cortés \& Hernández (2012) "El auditor ha sido vinculado casi en exclusividad a desempeñarse en lo relacionado a preparación, análisis, revisión y validación de estados financiero" por lo tanto se requieren de conocimientos, capacidades y cualidades la cuales varias entre cada auditor que desempeña el cargo.

No obstante los auditores son humanos tienden a cometer fallos provocando que según Rodriguez \& Gutierrez (2016) "muchas veces se vean cuestionados por no alcanzar los estándares de calidad que sus clientes desean, bien sea por el desconocimiento de sus responsabilidades y la falta de aplicación de los procedimientos necesarios." Esto es debido a que los auditores normalmente presentan dificultades en ciertas áreas durante la aplicación de la Auditoria en la entidad.

Según lo anterior, se presenta la siguiente incógnita, ¿Cuál es el perfil que debe tener un contador público para desarrollar una auditoría financiera? Esto debido a que la auditoria no la puede desempeñar cualquier contador, es por ello que el objetivo del presente artículo es determinar que capacidades y cualidades demandadas por el mercado, deberá cumplir el perfil de un contador público, para el ejercicio de una auditoría financiera.

\section{Materiales y métodos}

Para la localización de los documentos bibliográficos se utilizaron diversas fuentes documentales, se realizó una búsqueda bibliográfica en google académico, utilizando los descriptores: Cualidades del Auditor, Perfil de Auditoria, Auditoria Financiera, el Contador y la Auditoria, de esta forma consiguiendo la información necesaria tras la combinación de las diferentes palabras clave. Se seleccionaron aquellos documentos que contribuyeran en el desarrollo del artículo en aspectos tales como definiciones, cualidades, propiedades, características entre otros.

\section{Aproximaciones teóricas a la Auditoría Financiera}

Para iniciar primero debemos de conocer que es una auditoría financiera, la cual Nuño (2017) la define como "un método por el que se examina y analiza la información que una empresa tiene reflejada en los estados de sus cuentas." De esta manera dar una opinión acerca de que, si los estados financieros son elaborados fielmente a las normas de contabilidad, o si existen incorreciones materiales.

Este proceso debe ser realizado exclusivamente por un contador público o una firma de auditores externo a la empresa, no obstante, según Isotools (2017) el auditor deberá "de disponer de todos los conocimientos necesarios, contar con la experiencia necesaria y disponer de unas cualidades personales específicas." Con el propósito de disminuir los riesgos de errores durante el desarrollo de la auditoria las cuales pueden traer al Auditor una serie de inconvenientes. 
Por lo tanto, con el propósito de que el auditor disminuya el riesgo de cometer errores durante el proceso de auditoría, el auditor o equipo auditor deberá de contar con "perfiles profesionales multidisciplinarios con competencias técnicas y habilidades necesarias, y de esta forma contribuir con la generación de valor" (Mallen \& Collins, 2003) de esta manera el auditor tenga claridad del funcionamiento del negocio.

\section{Perfil del auditor}

Son varios los aspectos que debe de tener el perfil de un contador público para el desarrollo de una auditoría financiera, de esta forma, se tiene que un auditor según Frett (2014) "debe tener una actitud imparcial y neutral, para cualquier conflicto de intereses. La objetividad es una actitud mental neutral que permite a los auditores internos desempeñar su trabajo" con esta cualidad el auditor tendrá confianza del producto de la revisión de los estados financieros una vez finalizada la auditoria.

Otra cualidad destacable, es el hecho de que el auditor debe de guardar total confidencialidad con el cliente, es decir, no divulgar la información de la empresa a terceros debido a que esa información solo le pertenece a él, además, se podrían generar actos de mala fe, no obstante, según Alcaldía de Bogotá (2018) "si bien mantener una relación confidencial con el cliente, se vuelve fundamental, esto no debe ser motivo para que los profesionales se conviertan en cómplices de actos ilícitos." Los cuales son normalmente cometidos por organizaciones que buscan generar beneficios económicos sin dificultad alguna.

Además de lo anterior, un aspecto importante que debe poseer el auditor ante su cliente es la diplomacia total, es decir, saber tratar con las personas, el saber comunicarse ante sus clientes es una cualidad eficaz durante el desarrollo de cualquier auditoria, debemos tener en cuenta que auditado mantiene una preocupación constante por el hecho de que se le inspeccionen sus estados financieros.

Por otra parte, según Blum (2004) "aunque se actúe de manera responsable y ética, algunas decisiones tomadas por el auditor pueden no ser populares, pudiendo llegar a causar desacuerdos y confrontaciones." Provocando que en algunos casos el auditado decida negociar con el auditor para cambiar o eliminar algunas inconformidades del informe final, desvirtuando la eficacia del mismo, es por ello que el auditor deberá de ser firme ante sus decisiones al finalizar el proceso de auditoría.

Además, se tiene que el auditor deberá de poseer un pensamiento crítico ya que según Navarrete (2016) "este tipo de razonamiento requiere salirse de los propios juicios y prejuicios para considerar todas las perspectivas, cuestionar la validez de cada uno y llegar a una conclusión." De esta forma al analizar la información suministrada por el auditado tenga la capacidad de realizar un informe de auditoría eficaz, teniendo en cuenta que esta cualidad se desarrolla por medio de la retroalimentación y en el trabajo.

Así mismo, el auditor deberá ser independiente, es decir, que el auditor sea autónomo en el desempeño de sus funciones con el propósito de realizar su trabajo libremente sin que terceros interfiera para manipular el desarrollo de la auditoria, además, "La independencia le permite al auditor juzgar de manera imparcial y evaluar adecuadamente los estados financieros, la administración y el control interno de la empresa." (Auditool, 2017)

\section{Conclusiones}

Un contador público que desea desempeñarse como un auditor en una determinada organización deberá de tener en su perfil ciertos aspectos, cualidades y 


\section{6}

características, destacando; la independencia, la diplomacia, la imparcialidad, el pensamiento crítico y la confidencialidad, debido a que estas le permiten al auditor desarrollar el proceso de auditoría sin la influencia de terceros de esta manera elaborar un informe de auditoría sin adulteración alguna.

\section{Referencias}

Alcaldía de Bogotá. (2018). Código de ética del auditor interno de la Secretaría General. Registro Distrital, 51(6337), 1-3

Álvarez O. C. A. (2015). Guía para la elaboración de papeles de trabajo en auditoria financiera. Bogotá: Ediciones Universidad Cooperativa de Colombia. Obtenido de http://dx.doi. org/10.16925/greylit.1144

Auditool. (2017). El auditor externo independiente. Cómo garantizar la integridad, objetividad $e$ independencia, y evitar los conflictos de intereses. Obtenido de Auditool: https://www. auditool.org/blog/auditoria-externa/4822el-auditor-externo-independiente-comogarantizar-la-integridad-objetividad-eindependencia-y-evitar-los-conflictos-deintereses\#: : text $=\mathrm{La} \% 20$ integridad $\% 20$ requiere $\% 20$ que $\% 20$ el,violaci $\% \mathrm{C} 3 \% \mathrm{~B} 3 \mathrm{n} \% 20$ $\mathrm{de} \% 2010 \mathrm{~s} \% 20$

Blum, X. M. (2004). Las cualidades de un buen auditor. Obtenido de Marmolblum: https:// marmolblum.wordpress.com/2016/08/24/lascualidades-de-un-buen-auditor/

Frett, N. (2014). Barreras que Limitan la Objetividad de un Auditor Interno. Obtenido de Auditool: https://www.auditool.org/ blog/auditoria-interna/2473 - barreras que-limitan-la-objetividad-de-un-auditorinterno\#: : text $=$ Los $\% 20$ auditores $\% 20$ internos $\% 20$ deben $\% 20$ tener,el $\% 20$ producto$\% 20 \mathrm{de} \% 20$ su $\% 20$ revisi $\% \mathrm{C} 3 \% \mathrm{~B} 3$ n.
Gonzales D, E.E., Alfaro C, M. I., Cortés I, P. d., \& Hernández V, S. A. (2012). Competencias requeridas por el contador auditor, en función a los cambios experimentados por la profesión. Capic Review, 10, 53-62.

Isotools. (12 de JUN de 2017). ¿Cuáles son las cualidades que debe tener un auditor ISO 27001? Obtenido de ISOTools: https://www. isotools.com.mx/cuales-las-cualidades-auditoriso-27001/

Mallen, D., Collins, C.. (2003). Manual de habilidades para auditoría: una introducción básica a la planificación y realización de auditorías de sistemas de gestión en las organizaciones. Bogotá. ICONTEC

Navarrete Esparza, M., (2016). Propuesta de mejoramiento en las capacidades, habilidades humanas y conocimientos básicos del Contador Público y auditor en la Universidad del BíoBío. Tesis de pregrado, Universidad del BioBio

Nuño, P. (2017). Auditoria Financiera. Obtenido de Emprendepyme: https://www.emprendepyme. net/auditoria-financiera.html

Rodriguez R. J. C., \& Gutierrez, E. L. (2016). Competencia del contador publico auditor externo frente al control de calidad en la audtoria de estados financieroS (NIA 220). Villacicencio, Colombia. Obtenido de Repositorio Institucional: http://repository.ucc. edu.co/handle/ucc/12087 